Bundesgesundheitsbl - Gesundheitsforsch Gesundheitsschutz 2002 • 45:894-904 DOI 10.1007/s00103-002-0521-1

Originalien und Übersichtsarbeiten

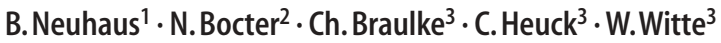

${ }^{1}$ Landesinstitut für den Öffentlichen Gesundheitsdienst (lögd) NRW Standort Münster

${ }^{2}$ Akademie für Öffentliches Gesundheitswesen, Düsseldorf

${ }^{3}$ Nationales Referenzzentrum für Staphylokokken am Robert Koch-Institut

\title{
Studie zum Vorkommen von Methicil- lin-resistenten Staphylococcus aureus in Alten- und Altenpflegeheimen in Nordrhein-Westfalen
}

\section{Zusammenfassung}

Anlass für diese Studie war die weltweite Zunahme von MRSA-Infektionen im Krankenhaus und der damit verbundenen Frage über das Vorkommen von MRSA in Altenund Altenpflegeheimen. Von März 2000 bis März 2001 wurde von 21 Gesundheitsämtern in Nordrhein-Westfalen insgesamt von 1057 Altenheimbewohnern und 193 Pflegekräften auf freiwilliger Basis Untersuchungsmaterial gewonnen und mit dem ausgefüllten Fragebogen an das lögd NRW weitergeleitet. In dieser Querschittsstudie zeigten 32 Bewohner und eine Beschäftigte einen positiven MRSA-Befund. Dies entspricht einer MRSA-Prävalenz von 3,0\% mit einem Konfidenzintervall (95\% KI) von 2,1-4,2, wenn als Bezugsgröße die Teilnehmerzahl benutzt wird, wie dies bisher bei MRSA-Untersuchungen in Altenheimen in Deutschland üblich war. Wird aber der S.-aureus-Anteil von durchweg fast $50 \%$ als Bezugsgröße zugrunde gelegt - wie dies bei MRSA-Untersuchungen in Krankenhäusern geschieht -, steigt die MRSA-Prävalenz auf das Doppelte, nämlich auf 6,3\% mit einem $\mathrm{KI}$ von 4,3-8,8. Das Tragen eines Katheters, insbesondere eines Harnwegskatheters, bedeutet ein fast 3-fach höheres Risiko, einen MRSA zu aquirieren $(\mathrm{OR}=2,72$, signifikant). Die MRSA-Prävalenz für Harnwegskatheter betrug $11,2 \%$ bezogen auf die S.-aureus-positiven Bewohner.

\section{Schlüsselwörter}

MRSA · Altenheime · Altenpflegeheime · Risikofaktoren · Präventionsstrategien · Hygieneempfehlungen
S. aureus ist ein Bakterium, das als wichtiger Verursacher von Infektionen innerhalb und außerhalb des Krankenhauses anzusehen ist. Er besiedelt allerdings auch die Haut des gesunden Menschen, wobei hier vorrangig die vordere Nasenhöhle kolonisiert wird. Ausgehend vom Nasenvorhof kann der Erreger sich auch auf den Rachen ausbreiten, auf die Hände, Achselhöhle, Analregion etc. übertragen werden. Bei abwehrgeschwächten Menschen können Staphylokokken, insbesondere S. aureus, schwere Infektionen verursachen wie Wundinfektionen, Lungenentzündung und Sepsis. Den gleichen Ausbreitungsweg kann die multiresistente oder Methicillin-resistente Variante von S. aureus - MRSA - nehmen. MRSA verursachen die gleichen Krankheiten wie S. aureus, nur dass sie wegen ihrer Antibiotikaresistenz wesentlich schwieriger zu behandeln sind, da nur noch wenige Antibiotika zur Behandlung in Frage kommen, die dann auch noch möglicherweise wegen ihrer Nebenwirkungen oder wiederum einsetzender Resistenzentwicklung nur begrenzt angewendet werden können.

Die Zunahme von MRSA-Infektionen stellt weltweit ein immer drängenderes Problem in stationären medizinischen Einrichtungen dar. Neben Ländern mit kaum noch beherrschbarer MRSASituation wie Großbritannien, USA und Japan oder Staaten aus Südeuropa, die einen Anteil von $30-80 \%$ an MRSAStämmen aufweisen $[1,2,3,4]$, sind die Länder - insbesondere die Niederlande und die skandinavischen Länder - her- vorzuheben, die infolge strikter Kontrollund Präventionsstrategien ihre MRSAAnteile auf unter $1 \%$ begrenzen konnten $[3,4]$. Für Deutschland lässt sich eine beunruhigende Zunahme des MRSAVorkommens in Krankenhäusern innerhalb von 8 Jahren von 1,7\% im Jahr 1990 über $12,9 \%$ im Jahr 1995 auf 15,2\% 1998 feststellen, bezogen auf die Anzahl der Patienten mit nachgewiesener S.-aureusBesiedlung oder -Infektion $[5,6]$.

Weltweit wird auch zunehmend über das Vorkommen von MRSA in Alten- und Altenpflegeheimen berichtet (Tabelle 1) [7,8,9,10,11,12,13,14].

Insbesondere aus den USA gibt es Daten über eine dramatische Zunahme der MRSA-Infektionen in Alten- und Altenpflegeheimen. Diese Einrichtungen werden als Reservoir für MRSA angesehen [15].

Es hat sich gezeigt, dass das Vorkommen von MRSA besonders an solche Heime gekoppelt ist, die mit Krankenhäusern der Maximalversorgung zusammenarbeiten. Es handelt sich aber in den meisten Fällen wohl um eine Besiedlung und nicht um eine Infektion. Dabei ist die Frage nur schwer zu klären, ob mehr Altenheimbewohner MRSA in die Krankenhäuser bringen oder mehr ent-

(c) Springer-Verlag 2002

Dr. Barbara Neuhaus

Landesinstitut für den öffentlichen Gesundheitsdienst (lödg), von Stauffenbergstr. 36, 48151 Münster, E-Mail: barbara.neuhaus@loegd.nrw.de 


\section{B. Neuhaus · N. Bocter · Ch. Braulke · C. Heuck $\cdot$ W. Witte}

Survey of methicillin-resistant Staphylococcus aureus in long-term facilities for the aged in northrhine westphalia

\section{Abstract}

The reason for conducting this study in North Rhine-Westphalia was the world-wide increase in MRSA hospital infections leading to the question about the occurrence of MRSA in old people's homes and nursing homes. Between March 2000 and March 2001,21 community health departments on a voluntary basis collected samples to be examined from a total of 1057 residents of old people's homes and 193 nursing staff members and, together with a completed questionnaire, sent them to the Institute of Public Health (lögd) NRW. In this cross-sectional study 32 residents and one staff member proved positive. This corresponds to a prevalence of MRSA of $3.0 \%$ with a confidence interval $(95 \% \mathrm{Cl})$ of $2.1-4.2$ if the number of participants is taken for reference as has until now been the usual practice in MRSA examinations in old people's homes in Germany. But if examinations are based on a Staph. aureus proportion of almost $50 \%$ as is done in MRSA examinations in hospitals, MRSA prevalence increases to $6.3 \%$ with a $\mathrm{Cl}$ of 4.3-8.8. Catheterization, particularly urinary catheterization, entails an almost three times higher risk of contracting MRSA (OR = 2.72 , significant). MRSA prevalence for urinary catheterization was $11.2 \%$, related to the S. aureus-positive residents.

\section{Keywords}

MRSA - old people's homes - risk factors prevention strategies · hygiene recommendations

Tabelle 1

Prävalenzen von MRSA in Pflegeheimen

\begin{tabular}{ll} 
Länder & MRSA-P \\
\hline USA [7] & $8,0-53$ \\
Großbritannien [8,9] & $4,7-17$ \\
Australien [10] & 11 \\
Niederlande [11] & $<1$ \\
Deutschland [12] & $0,0-2,9$
\end{tabular}

lassene Krankenhauspatienten MRSA in die Altenpflegeheime [16]. In Deutschland gibt es aus jüngster Zeit einige lokale Studien aus Berlin, Höxter, Köln, Frankfurt a.M. zum Vorkommen von MRSA in Alten- und Altenpflegeheimen. Sie werden vom Nationalen Referenzzentrum für Staphylokokken in Wernigerode $\mathrm{z}$. T. initiiert und zum Teil nur begleitet und ergaben einen MRSA-Anteil bis $\mathrm{zu} 2,9 \%$, bezogen auf die Altenheimbewohner [12,13,14].

Wegen der zunehmenden Bedeutung von MRSA in Krankenhäusern einerseits und den bisher spärlichen Kenntnissen auf diesem Gebiet in Altenheimen andererseits, hat das lögd NRW eine Studie zum Vorkommen von MRSA in nordrhein-westfälischen Alten- und Altenpflegeheimen durchgeführt, die dazu beitragen soll, Erkenntnisse über die Bedeutung von MRSA in diesen Einrichtungen zu bekommen und einige der noch offenen Fragen zu klären. Ein weiteres - inzwischen umgesetztes - Ziel dieser Studie war es, praxisorientierte Hygieneempfehlungen - auch in Hinblick auf das Vorkommen von MRSA - zu entwickeln, um die Hygiene in Alten- und Altenpflegeheimen zu stärken.

\section{Vorbereitung der Studie}

\section{Teilnehmer, Methode}

Im Juli 1999 stellte das Laborzentrum des Landesinstituts für den öffentlichen Gesundheitsdienst (lögd) NRW in Münster dem damaligen Ministerium für Arbeit, Gesundheit und Soziales NRW das Konzept einer Studie zur Frage nach der MRSA-Besiedlung von Bewohnern und Beschäftigten von Alten- und Altenpflegeheimen in NRW vor. Sowohl die Abteilung Gesundheit, die für die Gesundheitsämter zuständig ist, als auch die Abteilung Soziales, die die Aufsicht über die Altenheime hat, unterstützten diese Stu- die mit befürwortenden Anschreiben an alle Gesundheitsämter und Altenheimbetreiber in NRW. Somit waren die Gesundheitsämter vorbereitet, als das lögd mit einem ersten ausführlichen Anschreiben das Konzept vorstellte und um Teilnahme an dieser Studie bat. Es war nun Aufgabe der Gesundheitsämter, in den Altenheimen um Beteiligung zu werben. Bis Ende 1999 stand fest, dass sich 21 der insgesamt 54 Gesundheitsämter und 61 der insgesamt 1724 Altenheime in NRW beteiligen wollten. Insgesamt nahmen 1057 Bewohner und 193 Pflegekräfte auf freiwilliger Basis an der Studie teil. Es handelt sich um eine Querschnittsstudie. Bei der Entwicklung des Studiendesign und bei der mikrobiologischen Bestätigung der MRSA-Stämme hat das Nationale Referenzzentrum für Staphylokokken in Wernigerode mitgewirkt, bei der Entwicklung eines Anamneseerfassungsbogens und bei der Auswertung der Daten die Akademie für das öffentliche Gesundheitswesen in Düsseldorf.

Eine weitere Aufgabe der Gesundheitsämter bestand darin, die Einwilligungserklärungen von Bewohnern und ggf. von Betreuern und von Pflegekräften unterschreiben zu lassen, die anonymisierten Fragebögen auszufüllen bzw. ausfüllen zu lassen und die Abstriche zu gewinnen, und zwar Nasen- und Rachenabstriche, ggf. auch Wundabstriche. Die Einwilligungserklärungen blieben im Gesundheitsamt, Erfassungsbögen und Abstriche wurden schnellstmöglich an das lögd-Laborzentrum verschickt.

Bei der Auswahl der teilnehmenden Personen sollte folgendes beachtet werden:

D ca.je $1 / 3$ der teilnehmenden Bewohner sollten den 3 verschiedenen Pflegestufen angehören,

D die Anzahl der teilnehmenden Pflegekräfte sollte 10\% der Anzahl der Bewohner nicht übersteigen. 
Von März 2000 bis März 2001 wurden die Proben gewonnen und auf S. aureus und MRSA untersucht. Nach Eintreffen im Labor wurden die Abstrichtupfer aus ihrem Transportmedium überführt, in eine sterile Traubenzuckerbouillon und auf einen bluthaltigen Nähragar und Mannit-Kochsalz-Agar ausgestrichen und anschließend bei $36^{\circ} \mathrm{C}$ bebrütet. Nach 24 und 48 Stunden erfolgte die Beurteilung, ob S. aureus verdächtige Kolonien gewachsen waren. Im Verdachtsfalle wurden die Kolonien subkultiviert. Bei negativen festen Nährmedien wurde die Bouillonkultur ausgeimpft auf Blutagar und Mannit-Kochsalzagar. Bei verdächtigen Kolonien wurde anschließend die Plasmakoagulase mittels Kaninchenplasma geprüft bzw. ein Latex-Agglutinationstest auf S. aureus durchgeführt. Im positiven Falle wurde die Oxacillin-Resistenz im Agardiffusionstest auf DST-Agar, der $2 \%$ Kochsalz enthielt, mit $1 \mu \mathrm{g}$ Oxacillin bei $35^{\circ} \mathrm{C}$ für 24 Stunden und mit $5 \mu \mathrm{g}$ Oxacillin bei $30^{\circ} \mathrm{C}$ für 48 Stunden geprüft. Zeigte sich für Oxacillin eine auch nur partielle Resistenz, wurde der Stamm zur Bestätigung und zur molekularbiologischen Typisierung ins Nationale Referenzzentrum für Staphylokokken nach Wernigerode geschickt. Dort wurde mittels Fragmentmusteranalyse der genomischen DNS nach Sma I-Verdau der Gesamtzell-DNS und Pulsfeldgelelektrophorese der MRSA-Epidemiestamm bestimmt. Die detaillierte Durchführung ist bei Witte [17] beschrieben. Die numerische Auswertung der Fragmentmuster erfolgte anhand des von Claus [18] beschriebenen Datenbanksystems. Sowohl der S.-aureusBefund als auch der MRSA-Befund wurden protokolliert, da sie für die spätere Auswertung benötigt wurden.

Erst nach Bestätigung des MRSABefundes durch das NRZ für Staphylokokken wurde das positive Ergebnis über die Gesundheitsämter mündlich und schriftlich den behandelnden Ärzten und den Altenheimen mitgeteilt und das weitere Vorgehen über notwendige Schutzmaßnahmen, Aufklärungsarbeit, Umgebungsuntersuchungen usw. besprochen. Außerdem wurden vom Gesundheitsamt Ermittlungen zum Wohnumfeld angestellt. Gleichzeitig wurden den Altenheimen die vom lögd erarbeiteten Informationen und Empfehlungen zur Verhütung der Weiterverbreitung von MRSA in Alten- und Altenpflegeheimen zur Verfügung gestellt. Die negativen Ergebnisse wurden erst am Schluss der Untersuchungsserie jedem Gesundheitsamt mitgeteilt. Die statistische Auswertung erfolgte nach Eingabe aller personenbezogenen Daten aus den Erfassungsbögen und nach Eingabe der Ergebnisse der Laboruntersuchung.

Daten zur Person bezogen sich auf:

D Alter, Geschlecht, Aufnahmedatum,

D Einzel- oder Mehrbettzimmer,

D Pflegestufe und Mobilität,

D Materialentnahme (Nase, Rachen etc.),

D Grundkrankheiten in Form

Ja/Nein für folgende Krankheiten:

- Diabetes, periphere/zerebrale Durchblutungsstörungen,

- Ekzem,

- HIV,

- Dialysepflichtigkeit,

- Krebs,

- Endoprothesenträger.

Risikofaktoren in Form Ja/Nein:

D akute Infektion,

D offene Wunde,

D Dekubitus,

D Ulcus cruris,

Intubation,

- Katheter,

D Antibiotika,

Zytostatika,

Dorticoide,

D Operation innerhalb der letzten 6 Monate.

Für Bewohner, die in den vergangenen 6 Monaten außerhalb waren, wurde gefragt:

Drankenhausaufenthalt,

D anderes Altenheim,

anderes Pflegeheim,

zu Hause.

Für Pflegekräfte wurde nach Auslandsaufenthalten evtl. mit beruflicher Tätigkeit gefragt.
Die Reihenfolge der Expositionen ist grundsätzlich wie in den Erhebungsbögen gewählt. Die Ergebnisse sind entsprechend angeordnet. Die erhobenen, anonymisierten Daten wurden in der Akademie für öffentliches Gesundheitswesen in einer EPI-Info-Datenbank gespeichert. Die Auswertungen wurden mit den Modulen ANALYSIS und STATCALC von EPI-Info durchgeführt. Es erfolgte die Berechnung der Odds-Ratio für die Stichprobe und die Schätzung des Konfidenzintervalls.

\section{Ergebnisse}

Von den insgesamt 1724 Alten- und Altenpflegeheimen in NRW haben 61, das sind 3,5\%, an dieser Studie teilgenommen. Die 1057 teilnehmenden Bewohner machen bei 155.376 Altenheimbewohnern insgesamt einen Prozentsatz von $0,7 \%$ aus (s. Tabelle 2).

Bei einer Zielpopulation von rund 160.00o Bewohnern in Alten- und Altenpflegeheimen in NRW und einer in der Literatur bisher angegebenen Prävalenz für Deutschland von 0-2,9\% (siehe Tabelle 1) haben wir für die zu erwartende Prävalenz zwischen $2,4-2,9 \%$ in der geplanten Studie einen Zufallstichprobenumfang von 895 bis 1075 errechnet (EPITABLESample). Gut ein Drittel aller Gesundheitsämter (GÄ) in NRW beteiligten sich an unserer Studie. Bei den Pflegekräften lässt sich ein Prozentsatz nicht berechnen, da die Anzahl der in der stationären Pflege Tätigen nicht bekannt ist.

Die Angaben über Geschlecht, Altersgruppen, Pflegestufen und Dauer des Heimaufenthaltes sind in der nachfolgenden Tabelle 3 zusammengestellt. Daten über Art der Unterbringung, die Verteilung der Mobilität und Voraufenthalte in anderen Einrichtungen in den letzten 6 Monaten listet die Tabelle 4 auf.

Dabei zeigte sich, dass deutlich mehr Frauen als Männer, nämlich knapp $77 \%$
Tabelle 2

Studienteilnehmer
Gesamt NRW

n

Altenheime

Bewohner

Gesundheitsämter
1724

15.376

54
Studienteilnehmer

n

61

1057

21
3,5

0,7

39,0 
Tabelle 3

Altersverteilung, Geschlecht, Pflegestufen und Dauer des Heimaufenthaltes der beteiligten Heimbewohner (BW)

\begin{tabular}{lll} 
Altersgruppe & Anzahl d. Personen & $\%$ BW \\
\hline$<60$ Jahre & 54 & 5,1 \\
$60-69$ & 89 & 8,4 \\
$70-79$ & 263 & 24,9 \\
$80-89$ & 459 & 43,4 \\
$>90$ & 192 & 18,2
\end{tabular}

\section{Geschlecht}

\begin{tabular}{lll}
\hline Frauen & 813 & 76,9 \\
Männer & 240 & 22,7 \\
unbekannt & 4 & 0,4 \\
Pflegestufen & & \\
\hline
\end{tabular}

\begin{tabular}{lll}
0 & 33 & 3,1 \\
1 & 329 & 31,1 \\
2 & 415 & 39,3 \\
3 & 279 & 26,4 \\
unbekannt & 1 & 0,1 \\
Heimaufnahme & & \\
\hline vor 1996 & & \\
1996 & 251 & 23,7 \\
1997 & 104 & 9,8 \\
1998 & 133 & 12,6 \\
1999 & 149 & 14,1 \\
2000 & 229 & 21,7 \\
2001 & 180 & 17,0 \\
unbekannt & 2 & 0,2 \\
& 9 & 0,9
\end{tabular}

Tabelle 4

Art der Unterbringung, Mobilität, Voraufenthalte in den letzten 6 Monaten der beteiligten Heimbewohner (BW)

\begin{tabular}{lll} 
Unterbringung & Anzahl d. Personen & $\%$ BW \\
\hline Einzelzimmer & 519 & 49,1 \\
Mehrbettzimmer 2 Personen und mehr & 537 & 50,8 \\
unbekannt & 1 & 0,1 \\
Mobilität & &
\end{tabular}

\begin{tabular}{lll}
\hline $\begin{array}{l}\text { mobil, vollständig } \\
\text { Rollstuhl, Gehhilfen } \\
\text { bettlägerig } \\
\text { unbekannt }\end{array}$ & 270 & 25,5 \\
Voraufenthalte in den letzten 6 Monaten & 629 & 59,5 \\
& 152 & 0,6 \\
\hline Krankenhaus & 6 & \\
Anderes Altersheim & 246 & 23,3 \\
Anderes Pflegeheim & 5 & 0,5 \\
Zu Hause & 4 & 0,4 \\
& 18 & 1,7
\end{tabular}

gegenüber knapp 23\%, an der Studie teilnahmen. Die Altersgruppen der 70 - bis 89-Jährigen waren am häufigsten vertreten, insbesondere bei den Frauen; bei letzteren auch noch zusätzlich die Gruppe der über 90-Jährigen. Die Zugehörigkeit zu den 3 verschiedenen Pflegestufen war ungefähr gleich, ebenso die Unterbringung in Einzel- bzw. Mehrbettzimmer. Die Mehrzahl der Bewohner benötigte Gehhilfen bzw. einen Rollstuhl, knapp 15\% war bettlägerig, nur 1/4 der Bewohner war vollständig mobil. Immerhin knapp ein 1/4 aller Bewohner musste in den letzten 6 Monaten das Krankenhaus aufsuchen.

Bei den meisten Bewohnern wurden Nasen- und Rachenabstriche entnommen, bei 52 Bewohnern wurden zusätzlich noch Abstriche von anderen Körperstellen insbesondere aus Ulcus cruris, Decubitus und anderen infizierten Wunden wie Kathetereinstichstellen usw. entnommen.

Bei den Grundkrankheiten (Tabelle 5) überwogen der Diabetes, periphere und cerebrale Durchblutungsstörungen, bei den Risikofaktoren (Tabelle 6) das Tragen eines Katheters, hier insbesondere eines Harnwegskatheters (s. Tabelle 6a).

Bei der Analyse der insgesamt 22 akuten Infektionen fiel auf, dass 8 Infektionen der oberen Luftwege, 4 Dermatitiden und 4 Harnwegsinfektionen auftraten. Die restlichen 6 Infektionen verteilten sich auf Candidainfektion, Enteritis, Hepatitis C, Bursitis. Die Analyse der insgesamt 43 Wunden zeigte, dass 23 an den Extremitäten lokalisiert waren und 9 Decubiti festgestellt wurden. Die übrigen Wunden betrafen Kathetereinstichstelle, Kniefixateur, Stirnwunde u. ä.. Zum Zeitpunkt der Untersuchung erhielten 39 Personen eine Antibiotika-, 4 eine Zytostatika- und 27 eine Corticoidtherapie.

\section{„MRSA-Prävalenz: 3\% der Altenheimbewohner"}

Nachfolgend wird auf die MRSA-Prävalenz bezogen auf die Gesamtzahl der Bewohner und bezogen auf die Anzahl der S.-aureus-Nachweise bei den Bewohnern eingegangen. Diese zwei verschiedenen Prozentberechnungen wurden gewählt, weil die MRSA-Nachweisanteile aus Krankenhäusern sich immer auf die Gesamtzahl der S.-aureus-Nachweise beziehen. Daher wurde zur besseren Vergleichbarkeit von MRSA-Nachweisantei- 
Tabelle 5

Art der Grundkrankheiten der Bewohner (BW)

\begin{tabular}{lll} 
Grundkrankheiten & Anzahl & $\%$ BW \\
\hline Diabetes & 295 & 27,9 \\
Ekzem & 52 & 4,9 \\
HIV & 0 & 0,0 \\
Dialysepflicht & 5 & 0,5 \\
Krebs & 50 & 4,7 \\
Periphere Durchblutungsstörung & 201 & 19,0 \\
Zerebrale Durchblutungsstörung & 438 & 41,4 \\
Endoprothesenträger & 45 & 4,3 \\
Andere & 60 & 5,7
\end{tabular}

Tabelle 6

Risikofaktoren der Bewohner (BW)

\begin{tabular}{lll} 
Risikofaktoren & Anzahl & \% BW \\
\hline Akute Infektionen & 22 & 2,1 \\
Offene Wunden & 43 & 4,1 \\
Decubitus & 28 & 2,6 \\
Ulcus cruris & 14 & 1,3 \\
Intubation & 0 & 0,0 \\
Katheter & 197 & 18,6 \\
Antibiotika & 39 & 3,7 \\
Zytostatika & 4 & 0,4 \\
Corticoide & 27 & 2,6 \\
Operation innerhalb der letzten 6 Monate & 20 & 1,9
\end{tabular}

Tabelle 6a

Risikofaktor Katheter bezogen auf die Gesamtzahl der Bewohner (BW)

\begin{tabular}{lll} 
Katheter & $\mathbf{n}$ & $\begin{array}{l}\text { \% d. BW } \\
18,6\end{array}$ \\
\hline - Harnweg & 197 & 14,9 \\
- i.v. & 158 & 0,2 \\
- PEG & 2 & 6,8
\end{tabular}

len in Altenheimen zu den Anteilen in Krankenhäusern auch die S.-aureusAnzahl als Bezugsgröße gewählt. Bei den wenigen bisher erschienenen Arbeiten zu MRSA-Besiedlungsanteilen in Altenheimen war die Bezugsgröße immer die Bewohnerzahl.

In Tabelle 7 ist in der Übersicht der S.-aureus-Anteil mit Prozentangaben bezogen auf die Bewohnerzahl und auf das Pflegepersonal. Die MRSA-Anteile sind bezogen auf die Bewohnerzahl und die Gesamtzahl der teilnehmenden Pflegekräfte sowie auf die S.-aureus-positiven Bewohner und Pflegekräfte. Benutzt man als Bezugsgröße die teilnehmende Bewohnerzahl, so kommt man auf einen MRSAAnteil von 3,0\% mit einem KI von 2,1-4,2. Wird aber der S.-aureus-Anteil von fast
50\% zugrunde gelegt, so steigt die MRSAPrävalenz auf das Doppelte, nämlich auf $6,3 \%$ mit einem $\mathrm{KI}$ von 4,3-8,8 an.

Ähnlich sieht es beim Pflegepersonal aus, allerdings auf deutlich niedrigerem Niveau: der S.-aureus-Anteil beträgt ebenfalls fast $50 \%$, so dass die MRSAPrävalenz von 0,5 bei Zugrundelegung der Gesamtzahl der teilnehmenden Pflegekräfte auf 1,1 steigt, wenn die S.-aureusTräger unter den Pflegekräften als Bezugsgrößen dienen. Die insgesamt 33 MRSA-positiven Befunde wurden 31-mal aus Nasenabstrichen,30-mal aus Rachenabstrichen und aus 3 Wundabstrichen erhoben (Tabelle 8). In Tabelle 8 ist außerdem die unterschiedliche MRSAPrävalenz ersichtlich, je nach dem ob die Bewohnerzahl oder der S.-aureus-
Besiedlungsanteil (fast $50 \%$ ) zugrunde gelegt wird. Wird S. aureus als Bezugsgröße herangezogen, liegt die MRSAPrävalenz z.B. im Nasenabstrich bei 3,0\%; wird die Bewohnerzahl als Bezugsgröße angewendet, ist die MRSA-Prävalenz mehr als doppelt so groß, also 6,6\%.

In den Tabellen 9 und 10 sind MRSA- und S.-aureus-Besiedlungen nach Geschlecht, Alter Pflegestufe, Dauer des Heimaufenthalts und Mobilität, der Unterbringung und Voraufenthalte bezogen auf die Gesamtzahl der Bewohner (BW) und bezogen auf die S.aureus-Besiedlungen der Bewohner (S. au. BW) zu sehen. Daraus ist Folgendes ersichtlich: der Anteil der MRSA-Besiedlung bei Männern ist größer als bei Frauen. Die 60- bis 69-Jährigen und die 80- bis 89-Jährigen haben einen höheren MRSA-Besiedlungsanteil - unabhängig vom Geschlecht - als die übrigen Altersgruppen. Bei den Pflegestufen war erwartungsgemäß die Pflegestufe 3 diejenige mit dem höchsten prozentualen MRSA-Besiedlungsanteil. Die Dauer des Heimaufenthaltes spielte insofern eine Rolle, als bei längerem Heimaufenthalt höhere MRSA-Besiedlungen auftreten.

Bei eingeschränkter Mobilität zeigen sich höhere MRSA-Prävalenzen von 7,2\% bei Bewohnern mit Gehhilfen bzw. Rollstuhl und $7,9 \%$ bei bettlägrigen $\mathrm{Be}$ wohnern. In Mehrbettzimmern ist kein höherer MRSA-Anteil gegenüber Einbettzimmern $\mathrm{zu}$ finden. Auffallend in der Rubrik Voraufenthalte in den letzten 6 Monaten ist das Ergebnis, dass ein Krankenhausvoraufenthalt einen deutlich höheren MRSA-Besiedlungsanteil zeigt, und zwar besonders augenscheinlich, wenn der Wert von 13,1\% betrachtet wird, der unter Berücksichtigung der S.-aureusBesiedlung der Bewohner entstanden ist. Die übrigen Voraufenthalte spielten in Bezug auf MRSA-Besiedlung keine Rolle.

In Tabelle 11 sind die Grundkrankheiten der Heimbewohner aufgelistet mit den dazugehörigen MRSA- und S.aureus-Besiedlungsanteilen. Bei Grundkrankheiten wie periphere Durchblutungsstörung, Endoprothesenträgertum und andere Grundkrankheiten (z.B. chronische Pankreatitis, Nekrose am Fuß, Zustand nach $\mathrm{Tbc}$ ) finden wir eher höhere MRSA-Besiedlungsanteile.

Decubitus, Ulcus cruris, Katheter, insbesondere Harnwegskatheter, die Einnahme von Antibiotika, eine Operation in den letzten 6 Monaten gehören zu den 
Tabelle 7

MRSA- und S.-aureus-Besiedlung/-Infektion von Altenheimbewohnern (BW) und Pflegekräften (P)

\begin{tabular}{lllllll} 
& \multicolumn{2}{c}{ Teilnehmer } & \multicolumn{2}{c}{ S.aureus positiv } & \multicolumn{2}{c}{ MRSA positiv } \\
& n & n & \% d. BW & n & \% d. BW & \% d.S. au. \\
\hline & 1057 & 506 & 47,9 & 32 & 3,0 & 6,3 \\
& $\mathbf{n}$ & $\mathbf{n}$ & $\%$ d.P & n & \% d.P & $\%$ d.S. au. \\
\hline P & 193 & 93 & 48,2 & 1 & 0,5 & 1,1
\end{tabular}

Tabelle 8

S.-aureus- und MRSA-Befunde in den verschiedenen Untersuchungsmaterialien bezogen auf die Gesamtzahl der Abstriche und bezogen auf den S.-aureusBesiedlungsanteil der Bewohner (S. au. BW)

\begin{tabular}{lllllll} 
U-Material & Anzahl & \multicolumn{2}{l}{ S.au.incl. MRSA } & \multicolumn{2}{l}{ MRSA } \\
& $\mathbf{n}$ & $\mathbf{n}$ & $\%$ & $\mathbf{n}$ & $\%$ d. Abstriche & \% d. S. au. BW \\
\hline Nasenabstrich & 1049 & 471 & 44,9 & 31 & 3,0 & 6,6 \\
Rachenabstrich & 1030 & 466 & 45,2 & 30 & 2,9 & 6,4 \\
Decubitus & $\mathbf{8}$ & 4 & 50,0 & 1 & 12,5 & 25,0 \\
Ulcus & 13 & 8 & 61,5 & 2 & 15,4 & 25,0 \\
Trachealsekret & 2 & 2 & 50,0 & 0 & 0,0 & 0,0 \\
Wunde & 27 & 12 & 44,0 & 0 & 0,0 & 0,0 \\
Ekzem & 2 & 1 & 50,0 & 0 & 0,0 & 0,0
\end{tabular}

Risikofaktoren, bei denen eher mit einer MRSA-Besiedlung $\mathrm{zu}$ rechnen ist (s. Tabelle 12,13), obwohl die Signifikanzberechnungen nur für das Risiko Harnwegskatheter positiv ausfiel. Hier liegt das Risiko, einen MRSA zu aquirieren, fast dreimal so hoch wie bei Bewohnern ohne Harnwegskatheter.

Unter Zytostatikatherapie (4-mal) oder Corticoidtherapie (27-mal) wurde in keinem Falle MRSA im Nasen-/ Rachenraum der Bewohner festgestellt. Die Analyse der Wunden zeigte, dass bei insgesamt 43 Wunden nur einmal MRSA im Nasen-/Rachenraum der Betroffenen nachweisbar war (MRSA-Prävalenz: 2,3\% bezogen auf 43 Bewohner bzw. 3,7 $\%$ bezogen auf $27 \mathrm{~S}$. aureus besiedelte Bewohner). Bei insgesamt 39 mit Antibiotika behandelten Bewohnern wurde 2-mal MRSA nachgewiesen. Die univariate Auswertung des Tragens eines Harnwegskatheters ergibt die Odds Ratio von 2,72 bei 95\%igem Konfidenzintervall von 1,31-5,67 (Tabelle 14).

Die Ergebnisse der molekularbiologischen Typisierung der MRSA-Stämme gehen aus Tabelle 15 hervor. Der häufigste Stamm war der Berliner Stamm, gefolgt vom Hannover Stamm und
Stamm Barnim. Diese Stammverteilung spiegelt das Bild in nordrhein-westfälischen Krankenhäusern wider. Die Sma IMakrorestriktionsmuster der meisten MRSA-Stämme sind in Abb. 1 dargestellt. Die geographische Verteilung der insgesamt ermittelten 33 MRSA-Stämme ist aus Abb. 2 ersichtlich.

Von den 193 untersuchten Pflegekräften war nur eine Person MRSA-positiv (s. Tabelle 16), die auch den in diesem Altenheim MRSA-positiven Bewohner betreute. Bei den übrigen Pflegekräften konnte MRSA nicht nachgewiesen werden.

\section{Diskussion}

Die Reduzierung von Methicillin-resistenten Staphylococcus-aureus-Stämmen ist ein wichtiges Ziel des Hygienemanagements im Krankenhaus. Bei Erforschung von MRSA stellte sich heraus, dass das Vorkommen von MRSA nicht auf die Krankenhäuser beschränkt ist, sondern dass dieser Stamm auch in anderen Gemeinschaftseinrichtungen vorkommt, nämlich in Alten- und Altenpflegeheimen, also in Einrichtungen, in denen alte Menschen mit vielen Grundkrankheiten, Risikofaktoren und mit
Abwehrschwäche eng zusammenwohnen und vom selben Pflegepersonal gepflegt werden. Ziel der vorliegenden Studie ist es, einen Überblick über die MRSA-Situation in Altenheimen in NRW zu bekommen, um daraus gegebenenfalls notwendige Konsequenzen abzuleiten.

In deutschen Krankenhäusern lag das MRSA-Vorkommen 1998 im Durchschnitt bei 15,2\% [13]. Die wenigen Untersuchungen an Bewohnern in Alten- und Altenpflegeheimen in Deutschland zeigten bisher eine MRSA-Prävalenz von maximal 2,9\% (s. Tabelle 17), wie in der überregionalen Studie des Robert KochInstitutes und in der Studie aus Frankfurt a. M. dargelegt [12,14]. Eine Ausnahme bildet die Untersuchung aus Hannover, in der anlässlich einer MRSA-Infektion in einem Alten- und Pflegeheim bei einer Umgebungsuntersuchung festgestellt wurde, dass 21\% bzw. 26\% der Bewohner mit MRSA besiedelt waren [19].

In Ländern wie USA und Großbritannien werden in Pflegeheimen Besiedlungsraten bis $\mathrm{zu} 53 \%$ gefunden (s. Tabelle 1) $[7,8,9]$. Im Vergleich zu diesen Ländern sind die Verhältnisse in deutschen Altenheimen also noch als günstig anzusehen. Nur in den Niederlanden liegt die MRSA-Prävalenz unter 1\%. In der vorliegenden Studie des lögd NRW liegt die MRSA-Prävalenz von 3,0\% (KI 2,1-4,2) bei bisher üblicher Berechnung MRSA bezogen auf die untersuchte Bewohnerzahl - fast in derselben Größenordnung wie die Ergebnisse der überregionalen RKI- und Frankfurt/M.Studie. Wenn man allerdings als Bezugsgröße die S.-aureus-Besiedlungen heranzieht, wie das auch bei MRSA-Prävalenzberechnungen im Krankenhaus geschieht, die bei der untersuchten Personengruppe bei fast 50\% liegt, verdoppelt sich die MRSA-Prävalenz, so dass sich im Durchschnitt eine MRSA-Prävalenz von $6,3 \%$ (KI 4,3-8,8) errechnet.

Auch dieser MRSA-Anteil erscheint noch vergleichsweise niedrig. Betrachtet man aber die verschiedenen Untergruppen, so errechnen sich MRSA-Prävalenzen von z. B. 11,2\% bei Harnwegskatheterträgern, von $15,4 \%$ bei Bewohnern mit Operationen in den letzten 6 Monaten oder von $16,7 \%$ an Ulcus cruris erkrankten Bewohnern. Das heißt, es werden durchaus MRSA-Prävalenzen wie im Krankenhaus erreicht, immer vorausgesetzt, dass als Bezugsgröße die S.-aureus-Besiedlungen der Bewohner 
Tabelle 9

MRSA- und S.-aureus-Besiedlung des Nasen-/Rachenraumes von Bewohnern. Abhängigkeit vom Geschlecht, Alter, Pflegestufe und Dauer des Heimaufenthaltes bezogen auf die Gesamtzahl der Bewohner ( $B W=1057)$ und bezogen auf den S.-aureus-Besiedlungsanteil der Bewohner (S. au. BW)

\begin{tabular}{|c|c|c|c|c|c|}
\hline Merkmal & $\begin{array}{l}\text { Anzahl } \\
\text { n }\end{array}$ & $\begin{array}{l}\text { S. au. incl. MRSA } \\
\mathbf{n}\end{array}$ & $\begin{array}{l}\text { MRSA } \\
\text { n }\end{array}$ & $\begin{array}{l}\text { MRSA } \\
\% \text { d. BW }\end{array}$ & $\begin{array}{l}\text { MRSA } \\
\% \text { d.S.au. BW }\end{array}$ \\
\hline \multicolumn{6}{|l|}{ Geschlecht } \\
\hline Frauen & 813 & 388 & 23 & 2,8 & 5,9 \\
\hline Männer & 240 & 118 & 9 & 3,8 & 7,6 \\
\hline unbekannt & 4 & 1 & 0 & 0,0 & 0,0 \\
\hline \multicolumn{6}{|c|}{ Altersgruppe } \\
\hline$<60 \mathrm{~J}$ & 54 & 29 & 2 & 3,7 & 6,9 \\
\hline $60-69 \mathrm{~J}$ & 89 & 38 & 6 & 6,7 & 15,8 \\
\hline $70-79 \mathrm{~J}$ & 263 & 140 & 4 & 1,5 & 2,9 \\
\hline $80-89 \mathrm{~J}$ & 459 & 207 & 15 & 3,3 & 7,2 \\
\hline$>90 \mathrm{~J}$ & 192 & 93 & 5 & 2,6 & 5,4 \\
\hline unbekannt & 0 & 0 & 0 & 0,0 & 0,0 \\
\hline \multicolumn{6}{|l|}{ Pflegestufe } \\
\hline 0 & 33 & 16 & 2 & 6,1 & 12,5 \\
\hline 1 & 329 & 138 & 6 & 1,8 & 4,3 \\
\hline 2 & 415 & 212 & 10 & 2,4 & 4,7 \\
\hline 3 & 279 & 140 & 14 & 5,0 & 10,0 \\
\hline unbekannt & 1 & 1 & 0 & 0,0 & 0,0 \\
\hline \multicolumn{6}{|c|}{ Heimaufnahme } \\
\hline vor 1996 & 251 & 124 & 9 & 3,6 & 7,3 \\
\hline 1996 & 104 & 56 & 5 & 4,8 & 8,9 \\
\hline 1997 & 133 & 68 & 4 & 3,0 & 5,9 \\
\hline 1998 & 149 & 75 & 3 & 2,0 & 4,0 \\
\hline 1999 & 229 & 98 & 10 & 4,4 & 10,2 \\
\hline 2000 & 180 & 83 & 1 & 0,6 & 1,2 \\
\hline 2001 & 2 & 0 & 0 & 0,0 & 0,0 \\
\hline unbekannt & 9 & 3 & 0 & 0,0 & 0,0 \\
\hline
\end{tabular}

Tabelle 10

MRSA - und S.-aureus-Besiedlung des Nasen-/ Rachenraumes von Bewohnern in Abhängigkeit von der Mobilität, Art der Unterbringung, Voraufenthalte in den letzten 6 Monaten bezogen auf die Gesamtzahl der Bewohner (BW = 1057) und bezogen auf den S. aureus-Besiedlungsanteil der Bewohner (S.au. BW)

\begin{tabular}{|c|c|c|c|c|c|}
\hline Merkmal & $\begin{array}{l}\text { Anzahl } \\
\mathrm{n}\end{array}$ & $\begin{array}{l}\text { S. au. incl. MRSA } \\
\mathrm{n}\end{array}$ & $\begin{array}{l}\text { MRSA } \\
n\end{array}$ & $\begin{array}{l}\text { MRSA } \\
\% \text { d. BW }\end{array}$ & $\begin{array}{l}\text { MRSA } \\
\% \text { d.S.au. BW }\end{array}$ \\
\hline Mobilität vollständig & 270 & 124 & 4 & 1,5 & 3,2 \\
\hline Gehhilfen, Rollstuhl & 629 & 290 & 21 & 3,3 & 7,2 \\
\hline bettlägerig & 152 & 89 & 7 & 4,6 & 7,9 \\
\hline unbekannt & 6 & 4 & 0 & 0,0 & 0,0 \\
\hline \multicolumn{6}{|l|}{ Unterbringung } \\
\hline $\begin{array}{l}\text { Einzelzimmer } \\
\text { Mehrbettzimmer }\end{array}$ & 519 & 236 & 15 & 2,9 & 6,4 \\
\hline 2 Personen und mehr & 537 & 271 & 17 & 3,2 & 6,3 \\
\hline unbekannt & 1 & 0 & 0 & 0,0 & 0,0 \\
\hline \multicolumn{6}{|c|}{ Voraufenthalte in den letzten 6 Monaten } \\
\hline Krankenhaus & 246 & 122 & 16 & 6,5 & 13,1 \\
\hline Anderes Altenheim & 5 & 2 & 0 & 0,0 & 0,0 \\
\hline Anderes Pflegeheim & 4 & 2 & 0 & 0,0 & 0,0 \\
\hline Zu Hause & 18 & 3 & 0 & 0,0 & 0,0 \\
\hline
\end{tabular}

dient, wie dies auch bei der Berechnung von MRSA-Vorkommen im Krankenhaus üblich ist. Allerdings muss hier auch eine Einschränkung erfolgen. Ein genauer Vergleich mit KrankenhausMRSA-Prävalenzen ist insofern nicht möglich, als dort auch nicht die generellen S.-aureus-Besiedlungen ermittelt werden, sondern in der Regel nur die S.aureus- und MRSA-Infektionen.

Allerdings wird häufig auch im Krankenhaus nicht zwischen Infektion und Besiedlung unterschieden. Hinzu kommt, dass es sich bei Altenheimuntersuchungen auf MRSA in der Regel um eine Querschnittsstudie/Prävalenzstudie handelt. Die Erfassung von MRSA in einem Altenheim zu einem bestimmten Zeitpunkt erlaubt keine direkte Vergleichbarkeit mit MRSA-Infektionen bzw. -Besiedlungen im Krankenhaus. Trotzdem haben wir uns entschlossen, zumindest zusätzlich zu den MRSA-Prävalenzangaben, die sich auf die Bewohnerzahl beziehen, auch noch die MRSAPrävalenzangaben darzustellen, die sich auf die Anzahl der mit S. aureus besiedelten Bewohner beziehen, da sie eher mit den MRSA-Angaben aus Krankenhäusern vergleichbar erscheinen.

\section{Unterschiede in der MRSA-Prävalenz}

1. Die MRSA-Prävalenz liegt bei Männern etwas höher als bei Frauen (nicht signifikant).

2. In der Altersgruppe der 60- bis 69Jährigen und der 80 - bis 89 -Jährigen waren die MRSA-Prävalenzen deutlich erhöht (nicht signifikant).

3. Bei Bewohnern der Pflegestufe 3 zeigte sich erwartungsgemäß ein deutlich höherer MRSA-Anteil, als bei niedrigen Pflegestufen (nicht signifikant). Dem Wert von $12,5 \%$ in Pflegestufe o sollte wegen der geringen Fallzahl von nur 2 Personen keine große Bedeutung zugemessen werden.

4. Bei dem Charakteristikum Heimaufnahme ist ein Widerspruch vorhanden. Bewohner, die lange im Heim leben - hier seit 1996 und länger haben mit $8,9 \%$ und 7,3\% MRSA-Prävalenz ein höheres Risiko, als Bewohner, die erst seit 1998 im Altersheim wohnen. Möglicherweise bringt ein längerer Heimaufenthalt auch größeren Selektionsdruck im weitesten Sinne mit sich. Aber Menschen, die erst seit 1999 im Heim leben, weisen 


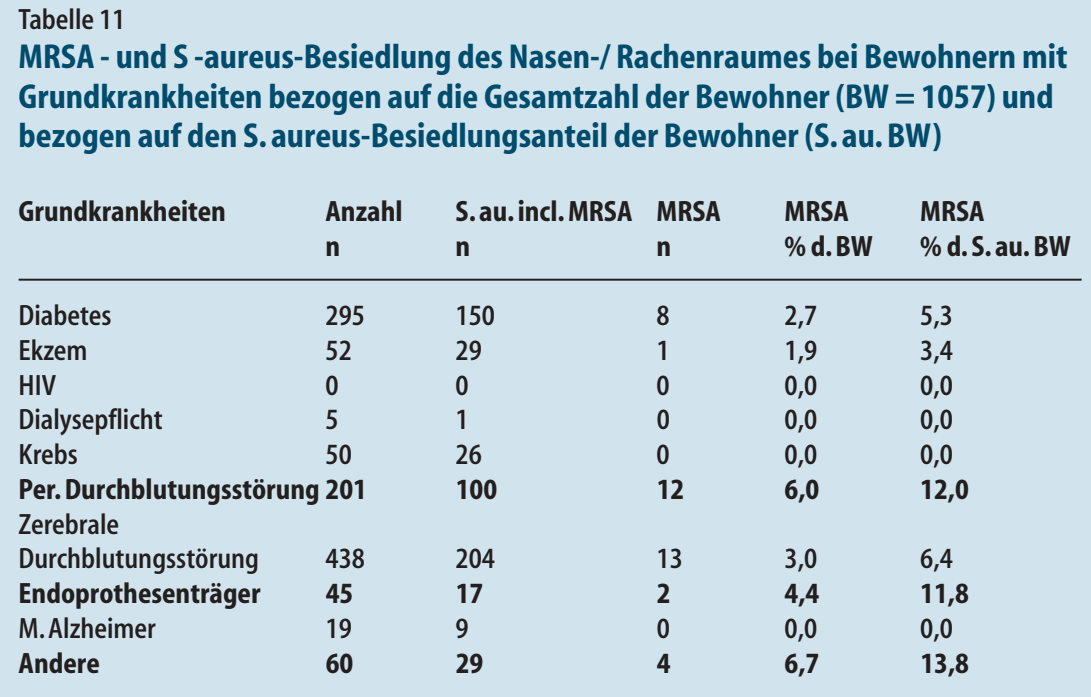

Tabelle 12

MRSA - und S.- aureus-Besiedlung des Nasen-/ Rachenraumes bei Bewohnern mit Risikofaktoren bezogen auf die Gesamtzahl der Bewohner ( $B W=1057)$ und bezogen auf den S. aureus-Besiedlungsanteil der Bewohner (S. au. BW)

\begin{tabular}{llllll} 
Risikofaktoren & $\begin{array}{l}\text { Anzahl } \\
\mathbf{n}\end{array}$ & $\begin{array}{l}\text { S. au. incl. MRSA } \\
\mathbf{n}\end{array}$ & $\begin{array}{l}\text { MRSA } \\
\mathbf{n}\end{array}$ & $\begin{array}{l}\text { MRSA } \\
\% \text { d. BW }\end{array}$ & $\begin{array}{l}\text { MRSA } \\
\% \text { d.S. au. BW }\end{array}$ \\
\hline Akute Infektionen & 22 & 13 & 1 & 4,5 & 7,7 \\
Offene Wunden & 43 & 27 & 1 & 2,3 & 3,7 \\
Decubitus & 28 & 19 & 2 & 7,1 & 10,5 \\
Ulcus cruris & 14 & 6 & 1 & 7,1 & 16,7 \\
Intubation & 0 & 0 & 0 & 0,0 & 0,0 \\
Katheter & 197 & 112 & 12 & 6,1 & 10,7 \\
Antibiotika & 39 & 21 & 2 & 5,1 & 9,5 \\
Zytostatika & 4 & 2 & 0 & 0,0 & 0,0 \\
Corticoide & 27 & 15 & 0 & 0,0 & 0,0 \\
Operation innerhalb & 20 & 13 & 2 & 10,0 & 15,4 \\
der letzten 6 Monate & & & & &
\end{tabular}

\section{Tabelle 13}

MRSA- und S.-aureus-Besiedlung des Nasen-/Rachenraumes bei Katheterträgern bezogen auf die Gesamtzahl der Bewohner (BW = 1057) und bezogen auf den S.-aureus-Besiedlungsanteil der Bewohner (S. au. BW)

\begin{tabular}{|c|c|c|c|c|c|}
\hline & $\begin{array}{l}\text { Anzahl } \\
\mathrm{n}\end{array}$ & $\begin{array}{l}\text { S. au. incl. MRSA } \\
\mathbf{n}\end{array}$ & $\begin{array}{l}\text { MRSA } \\
n\end{array}$ & $\begin{array}{l}\text { MRSA } \\
\% \text { d. BW }\end{array}$ & $\begin{array}{l}\text { MRSA } \\
\% \text { d.S.au. BW }\end{array}$ \\
\hline Katheter & 197 & 112 & 12 & 6,1 & 10,7 \\
\hline Harnweg & 158 & 89 & 10 & 6,3 & 11,2 \\
\hline i.v. & 2 & 0 & 0 & 0,0 & 0,0 \\
\hline PEG & 72 & 38 & 3 & 4,2 & 7,9 \\
\hline
\end{tabular}

eine noch höhere MRSA-Prävalenz $(10,2 \%)$ auf.

5. Bei geringer bzw. fehlener Mobilität (Gehhilfen, Rollstuhl, Bettlägerigkeit) liegt die MRSA-Prävalenz mit 7,2\% deutlich höher als bei mobilen

Bewohnern (3,2\%) (nicht signifikant).

6. Die MRSA-Prävalenz ist assoziiert mit dem Voraufenthalt in einem Krankenhaus. Sie beträgt in einem solchen Fall 13\% im Vergleich zur Durchschnitts- prävalenz von $6,3 \%$ (nicht signifi-

kant). Dieses Ergebnis macht deutlich, dass die Bewohner, die vorher in einem Krankenhaus lagen, in größerem Maße MRSA-besiedelt sind. Auch wenn dieses Ergebnis nicht signifikant ist, ist der Verdacht naheliegend, dass eher Altenheimbewohner MRSA aus dem Krankenhaus mitbringen, als umgekehrt MRSA ins Krankenhaus hineinbringen.

7. Bei Grundkrankheiten, wie periphere Durchblutungsstörungen, und das

Tragen von Endoprothesen zeigt sich häufiger eine MRSA-Besiedlung als ohne diese Grundkrankheiten. Die als andere Grundkrankheiten in Tabelle 11 bezeichneten Grundkrankheiten stellen eine Zusammenfassung verschiedener chronischen Infektionen dar.

8. Bei folgenden Risikofaktoren zeigt sich eine erhöhte MRSA-Prävalenz:

- akute Infektionen mit 7,7\%,

- Decubitus mit 10,5\%,

- Ulcus cruris mit $16,7 \%$,

- Harnwegskatheterträger mit 11,2\% signifikant,

- Antibiotikagabe mit 9,5\%,

- Operation innnerhalb der

letzten 6 Monate mit 15,4\%.

Das Risiko Harnwegskatheter ist als einziges signifikant. Alle übrigen Risiken, bei denen zwar eine Odds Ratio von über 1 gefunden wurde, sind aber nicht signifikant.

Keine Unterschiede bezüglich der MRSA-Prävalenzen ergaben sich bei der Unterbringung in Einzel- oder Mehrbettzimmern, bei Grundkrankheiten wie Diabetes, Ekzem, zerebrale Durchblutungsstörung, bei Risikofaktoren wie akute Infektion, offene Wunde.

Bei der überwiegenden Anzahl der MRSA-Nachweise handelte es sich um Einzelfälle jeweils pro Altenheim. Sechsmal allerdings kam es vor, dass in einem Altenheim 2 MRSA-positive Bewohner (BW) identifiziert wurden; in einem Altenheim waren ein Bewohner und eine Pflegekraft (P) MRSA-positiv mit demselben MRSA-Typ:

$1 \mathrm{x}$ Berliner Stamm bei 1 BW und $1 \mathrm{P}$, $1 \mathrm{x}$ Hannover Stamm bei 2 BW, $2 \mathrm{x}$ Barnim Stamm bei jeweils 2 x 2 BW, $1 \mathrm{x}$ MRSA nicht typisierbar bei 2 BW, $1 \mathrm{x}$ Süddeutscher Stamm bei 2 BW. 


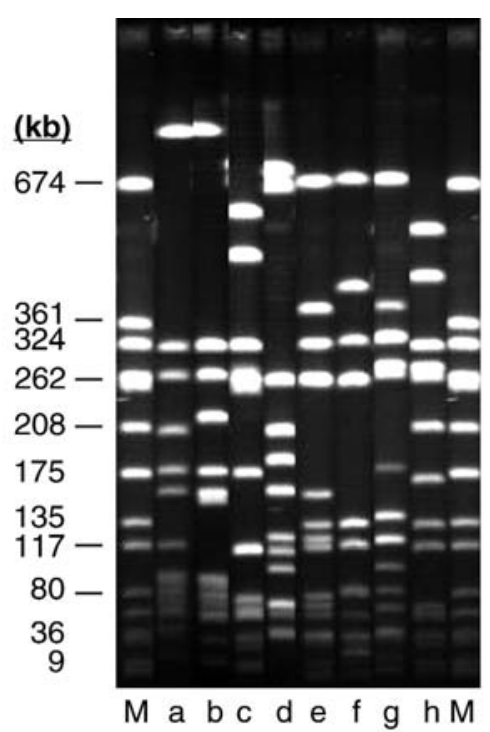

$\mathrm{M}=S$.aureus 8325; Mol.-Massen-Standard

$\mathrm{a}=$ Berliner Epidemiestamm

b $=$ Berliner Epidemiestamm Subklon

c = Süddeutscher Epidemiestamm Subklon

$\mathrm{d}=$ Barnim Epidemiestamm

$\mathrm{e}, \mathrm{f}=$ Beispiele für klassische MRSA

g = Subklon der Gruppe "Hannoverscher Epidemiestamm"

$\mathrm{h}=$ Rhein-Hessen-MRSA

Abb. $1 \Delta$ Beispiele für Smal Makrorestriktionsmuster von MRSA aus Besiedlung in Altenheimen

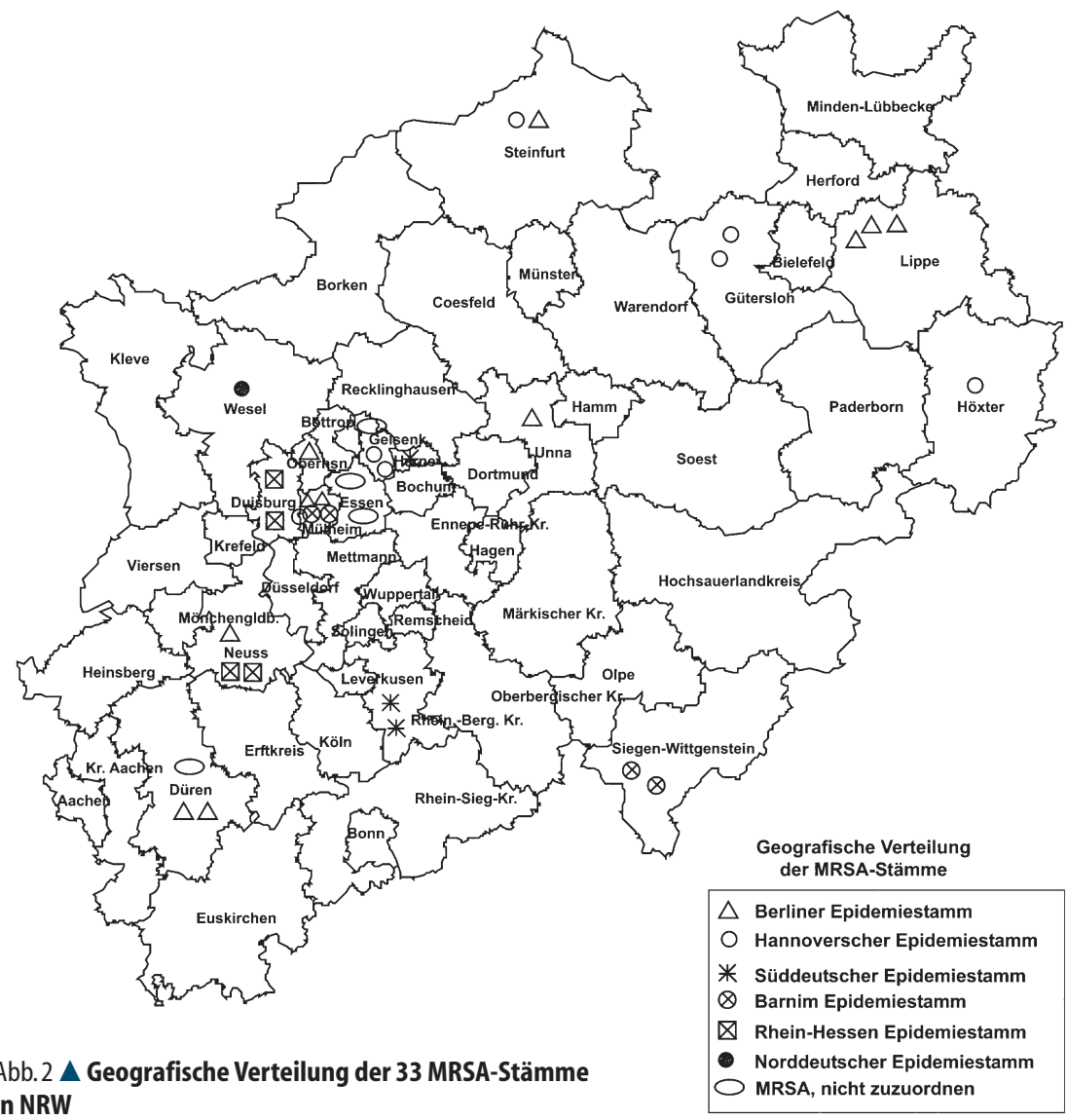

Tabelle 14

MRSA - Besiedlungsrisiken

Tabelle 15 MRSA-Epidemiestämmen
Berliner

Hannover

Barnim

MRSA, nicht differenzierbar

Rhein Hessen

Süddeutsch

Norddeutsch

Gesamt

\begin{tabular}{llll} 
Risikofaktoren & $\mathbf{O R}^{\mathbf{a}}$ & $\mathbf{K I}^{\mathbf{b}}$ & Signifikanz \\
\hline Decubitus & 2,56 & $0,58-11,29$ & nicht signifikant \\
Ulcus cruris & 2,51 & $0,32-19,8$ & nicht signifikant \\
Katheter (HW) & 2,72 & $1,31-5,67$ & signifikant \\
Antibiotika & 2,62 & $0,76-8,96$ & nicht signifikant \\
Op. in 6 Mon. & 3,73 & $0,83-16,8$ & nicht signifikant \\
\hline
\end{tabular}

${ }^{a} \mathrm{OR}=$ Odds Ratio, ${ }^{b} \mathrm{KI}=$ Konfidenzintervall

Zuordnung der isolierten MRSA zu den in Mitteleuropa bekannten

Stamm Bewohner Personal

11

11

7

4

4

3

1

32
1
In allen Fällen konnte mit Mupirocin-Nasensalben-Therapie eine Eradikation von MRSA erreicht werden. Eingeleitete Umgebungsuntersuchungen verliefen negativ. In keinem Heim kam es zur weiteren Ausbreitung von MRSA.

Nur in 3 Fällen zeigte sich eine echte Infektion mit MRSA. Bei 2 Bewohnern war ein Ulcus cruris mit MRSA infiziert, nur einer dieser Bewohner hatte gleichzeitig positive Nasen- und Rachenabstriche. Bei einem weiteren Bewohner war das Dekubitalgeschwür mit MRSA infiziert, gleichzeitig auch Nase und Rachen mit MRSA besiedelt. Nach Mupirocin-Nasensalbenbehandlung waren auch bei allen infizierten Bewohnern die Nasen-Rachen-Ergebnisse negativ. Die Sanierung machte nur bei dem Patienten mit Ulcus cruris am Bein Schwierigkeiten. Erst im Mai 2001 führte die lokale Behandlung mit desinfizierenden Maßnahmen bei diesem Patienten zur Abheilung.

In Übersicht 1 sind die Risikofaktoren noch einmal zusammenfassend dargestellt, die in nordrhein-westfälischen Altenheimen zu einer Kolonisation/ Infektion mit MRSA führen können. Sie entsprechen im Wesentlichen den Risikofaktoren für die Kolonisation mit 
Tabelle 16

MRSA- und S. aureus-Besiedlung bezogen auf die Untersuchungsmaterialien der Pflegekräfte (P)

\begin{tabular}{lllllll} 
& & & \multicolumn{3}{c}{ MRSA positiv } \\
Materialien & $\mathbf{n ~ P}$ & $\mathbf{n ~ S . a u . i n c l . M R S A}$ & $\%$ d.P & n & $\%$ P & \% S. au.P \\
\hline Nase & 193 & 93 & 48,2 & 1 & 0,5 & 1,1 \\
Rachen & 193 & 92 & 47,7 & 1 & 0,5 & 1,1
\end{tabular}

Tabelle 17

Vergleich der in unterschiedlichen Studien in Deutschland ermittelten Häufigkeiten der MRSA-Besiedlung/-Infektion in Alten- und Pflegeheimen

\begin{tabular}{|c|c|c|c|}
\hline $\begin{array}{l}\text { Bewohner/ } \\
\text { Heime }\end{array}$ & $\begin{array}{l}\text { MRSA- } \\
\text { Träger } \\
\mathbf{n}\end{array}$ & $\begin{array}{l}\text { MRSA-Prävalenz } \\
\text { in \% bei BW mit KI }\end{array}$ & $\begin{array}{l}\text { MRSA-Prävalenz } \\
\text { in \% bei S. au. - } \\
\text { BW mit KI }\end{array}$ \\
\hline
\end{tabular}

$\begin{array}{lllll}\text { Frankfurt/O., Brandenburg [12] } & 159 / 1 & 4 & 2,5 & - \\ \text { Berlin [13] } & 579 / 12 & 17 & 2,9 & - \\ \text { Höxter, NRW [13] } & 121 / 9 & 0 & 0,0 & - \\ \text { Köln, NRW [13] } & 82 / 1 & 1 & 1,2 & - \\ \text { Frankfurt/M., Hessen (14) } & 401 / 8 & 10 & 2,4 & - \\ \text { lögd-Studie, NRW } & 1057 / 61 & 32 & 3,0 & 6,3 \\ & & & \text { KI2,1-4,2 } & \text { KI 4,3-8,8 }\end{array}$

MRSA, die in der Literatur beschrieben sind $[12,14,16,17]$.

\section{Schlussfolgerungen}

Diese auf Freiwilligkeit beruhende Querschnittsstudie kann bei einem Stichprobenanteil von ca. $1 \%$ der Altenheimbewohner in NRW die MRSA-Situation landesweit in Alten- und Altenpflegeheimen repräsentativ beleuchten.

Es kann nach den Ergebnissen der Studie festgestellt werden, dass die MRSA-Prävalenzen in Alten- und Altenpflegeheimen nicht viel niedriger liegen als in Krankenhäusern, in besonderen Fällen sogar gleich hoch oder höher. Die Tatsache, dass bei 32 MRSA-Nachweisen nur 3 Bewohner mit MRSA infiziert waren, wovon nur einer bekannt war, die übrigen 29 also nur Besiedlungen darstellten und dass es in keinem Fall zu einer Weiterverbreitung über 2 Personen hinaus kam, spricht dafür, dass die bisher durchgeführten Hygienemaßnahmen ausreichten, um Kleinraumepidemien zu vermeiden.

\section{„Das Risikopotenzial für Infektionen durch MRSA in Altenheimen steigt."}

Die Morbidität und damit auch die Mortalität durch MRSA scheinen insgesamt in Altenheimen eher gering $\mathrm{zu}$ sein. Da der Anteil alter Menschen an der Gesamtpopulation aber zunimmt, damit auch die Multimorbidität und Pflegebedürftigkeit, steigt auch das Risikopotenzial für Infektionen durch MRSA in Alten- und Altenpflegeheimen. Daher sollten alle bei der Betreuung und Pflege von Menschen in Altenund Altenpflegeheimen Beschäftigten Kenntnisse über MRSA erwerben und MRSA-Präventionsstrategien beachten. Es muss versucht werden, durch die Einführung eines guten Hygienemanagements, vor allem durch Beachtung von Hygieneplänen, die multiresisten Bakterien und damit auch MRSA zurückzudrängen.

Dabei darf natürlich die Wohn- und Lebensqualität der Heimbewohner nicht leiden. Die Einführung der Ausbildung zum hygienebeauftragten Altenpfleger in NRW trägt dieser Situation Rechnung. Aber es muss auch zu intensivem Hygie- nebewusstsein der übrigen Altenpflegemitarbeiter kommen, was durch Schulung, Fortbildung und Thematisierung von Hygienefragen erreicht werden kann. Außerdem muss ein System in Altenheimen etabliert werden, das es ermöglicht, mit multiresistenten Erregern kolonisierte bzw. infizierte Patienten zu erfassen, auch wenn diese Forderung bedauerlicherweise im neuen Infektionsschutzgesetz (IfSG) keine gesetzliche Grundlage gefunden hat. Allerdings ist das gehäufte Auftreten von nosokomialen Infektionen auch aus Altenheimen an das Gesundheitsamt unverzüglich zu melden ( $\$ 6$ (3) IfSG). Außerdem sind nach $\$ 36$ IfSG in einem Hygieneplan innerbetriebliche Verfahrensweisen zur Infektionshygiene festzulegen, also auch Verfahrensweisen zum Umgang mit MRSA bzw. Präventionsmaßnahmen zur Vermeidung der Weiterverbreitung von MRSA.

\section{„Je effektiver die Hygienemaß- nahmen, desto niedriger sind die MRSA-Besiedlungen bzw. -Infektionen."}

In der Vorbereitungsphase der Studie wurde vom lögd auch eine Informationsschrift mit Empfehlungen zur Verhütung der Weiterverbreitung von MRSA in Alten- und Altenpflegeheimen entwickelt, in der auch ein Informationsblatt für betroffene Bewohner enthalten ist, analog den Empfehlungen des RKI [20], die dann auch jedem Altenheim, in dem MRSA nachgewiesen wurde, zur Verfügung gestellt wurde und zum Abschluss der Studie auch allen teilnehmenden Altenheimen und sonstigen Interessierten durch die Gesundheitsämter oder durch das lögd übergeben werden kann.

\section{Danksagung}

Den Altenheimen und Gesundheitsämtern, die sich an der Studie beteiligten, möchten wir für ihre Mühe sehr herzlich danken: Stadt Aachen, Kreis Aachen, Stadt Duisburg, Kreis Düren, Stadt Essen, Stadt Gelsenkirchen, Kreis Gütersloh, Stadt Hamm, Stadt Herne, Kreis Höxter, Kreis Lippe, Stadt Mülheim an der Ruhr, Stadt Münster, Kreis Neuss, Stadt Oberhausen, Kreis Recklinghausen, Rheinisch-Bergischer 


\section{Übersicht 1}

Risikofaktoren, die nach der lögd-Studie zu einer größeren Wahrscheinlichkeit der MRSA-Besiedlung des Nasen-/Rachenraumes von Bewohnern in Altenheimen führen

D Zunehmendes Alter,

D Abnehmende Mobilität,

D Hohe Pflegestufe,

D Geschlecht männlich,

Dangdauernde Antibiotikatherapie,

D Hospitalisierung in den letzten 6 Monaten,

D Periphere Durchblutungsstörung,

D Endoprothese,

Decubitus,

D Ulcus cruris,

D andere Grundkrankheiten (Pankreatitis, Fußnekrose, Zst.n. Tbc),

D längerer Heimaufenthalt,

Datheter, insbesondere Harnwegskatheter

Kreis, Kreis Siegen-Wittgenstein, Kreis Steinfurt, Kreis Unna, Kreis Wesel.

Außerdem gebührt Ulrike Schmidt vom lögd Dank für ihren unermüdlichen Einsatz bei der Organisation der Studie, bei der Durchführung der Laboruntersuchungen und bei der Auswertung der Ergebnisse.

\section{Literatur}

1. Voss A, Milatovic D, Wallrauch-Schwarz C, Rosdahl VT, Braveny I (1994) Methicillin-resistant Staphylococcus aureus in Europe. Eur I Clin Microbiol Infect Dis, 13 (1):50-55

2. Voss A, Kresken M (1996) Antibiotic resistance in staphylococci in Europe. Chemother, 42 (Suppl.2): 13-18

3. Konno M (1995) Nosocomial infections caused by methicillin-resistant Staphylococcus aureus in Japan.J Infect Chemother, 1: 30-39

4. Jarvis WR, Martone WJ (1992) Predominant pathogens in Hospital infections. J Antimicrob Chemother, 29 (Suppl A): 19-24

5. Nationales Referenzzentrum für Staphylokokken am RKI (Bereich Wernigerode) (2000) Zunahme des Auftretens von MRSA in deutschen Krankenhäusern. Epidemiologisches Bulletin 9,70-71

6. Kresken M, Hafner D, Studiengruppe (2000) Resistenzsituation bei klinisch wichtigen Infektionserregern gegenüber Chemotherapeutika in Mitteleuropa. Chemotherapie J, 9,51-86
7. Bradley SF (1999) Methicillin-resistant Staphylococcus aureus: long term care concerns. Am J Med; 106(5A), 2-10

8. Cox RA, Bowie PE (1999) Methicillin-resistant Staphylococcus aureus colonization in nursing home residents: a prevalence study in Northhamptonshire.J Hosp Infect; 43(2), 115-122

9. Fraise AP, Mitchell K, $O^{\prime} B$ rien SJ, Oldfield K, Wise R (1997) Methicillin resistant Staphylococcus aureus (MRSA) in nursing homes in a major UK city: an anonymized point revalence survey. Epidemiol Infect; 118(1), 1-5

10. Flint JA, Ryan P, Gordon DL (1998) Prevalence of MRSA in South Australian nursing homes. Med J Aust, 169(10), 559-560

11. Hoebe CJ (1999) Prevention of a suspected epidemic of methicillin-resistant staphylococcus aureus (MRSA) in a nursing home. Ned. Tijdschr Geneeskd, 143(19), 994-997

12. Heuck D, Claus H, Fell G, Roth H, Keine M, Brenner K-P,Witte W (1999) MRSA-Besiedlung bei Bewohnern von Alten- und Altenpflegeheimen: Erste Ergebnisse einer überregionalen Studie.51.Jahrestagung der Deutschen Gesellschaft für Hygiene und Mikrobiologie, 11.14.10.1999 in Regensburg

13. Heuck D, Fell G, Hamouda O, Claus H, Witte W (2000) Erste Ergebnisse einer überregionalen Studie zur MRSA-Besiedlung bei Bewohnern von Alten- und Pflegeheimen. Hygiene und Medizin 25, 191-192

14. Heudorf U (1999) MRSA-Besiedlung bei Bewohnern von Alten- und Pflegeheimen sowie bei Patienten einer geriatrischen Rehabilitationsklinik in Frankfurt a. M. 1999, Stadtgesundheitsamt Frankfurt a. M., Abteilung Umweltmedizin und Hygiene, Braubachstr. 1822,60311 Frankfurt a.M.

15. Muder RR, Brennen C, Wagener MM, Vickers RM, Rihs JD, Hancock GA, Yee YC, Miller JM, Yu VL (1991) Methicillin-resistent staphylococcal colonisation and infection in a long-term care facility. Ann. Intern Med 114, 107-112
16. Heuck D, Nassauer A (1999) Methicillin-resistente Staphylococcus aureus in Alten- und Altenpflegeheimen. Hyg Med 24,72-80

17. Witte W, Cuny C, Claus H (1993) Clonal relatedness of Staphylococcus aureus strains from infections in humans as deduced from genomic DNA fragment patterns. Med. Microb. Lett.2,72-79

18. Claus H, Cuny C, Pasemann B, Witte W (1998) A database system for fragment patterns of genomic DNA of Staphylococcus aureus. Zentralbl. Bakteriol.287, 105-116

19. Höpken M-E, Dreesmann J, Braulke Ch, Heuck D, Witte W (2001) MRSA-Besiedlung in einem Alten- und Altenpflegeheim: Risikofaktoren und Prävalenz. Hyg Med 26, 225-230

20. Mitteilung der Kommission für Krankenhaushygiene und Infektionsprävention am RKI (1999) Empfehlungen zur Prävention und Kontrolle von Methicillin-resistenten Staphylococcus aureus-Stämmen (MRSA) in Krankenhäusern und anderen medizinischen Einrichtungen. Bundesgesundheitsblatt;42: 954-958 